\title{
Craniectomía descompresiva primaria en la hemorragia subaracnoidea aneuris- mática. Resultados de un estudio piloto en 11 casos
}

\author{
F. Arikan; J. Vilalta; F.J. Romero*; I. Porta**; F.R. Martínez-Ricarte y J. Sahuquillo
}

Servicio de Neurocirugía, Unidad de Investigación de Neurotraumatología-Neurocirugía. Institut de Recerca Vall d’Hebron. Universidad Autónoma de Barcelona. Unidad de Neuroradiología Intervencionista* y Unidad de Cuidados Intensivos**. Hospital Universitario Vall d'Hebron, Barcelona.

Resumen

Introducción. A pesar de los avances científicos y técnicos de los últimos años, la hemorragia subaracnoidea aneurismática (HSAa) sigue presentando una alta morbilidad y mortalidad. Este hecho, junto con los impresionantes resultados de la craneotomía descompresiva primaria (CDP) en el infarto maligno de la arteria cerebral media sugiere la posibilidad que la CDP sea una alternativa terapéutica a considerar en algunos pacientes con una HSAa. Presentamos nuestra experiencia de un estudio piloto en el que se utilizó la CDP en pacientes con HSAa en mal grado neurológico y hematoma intracerebral asociado.

Pacientes y métodos. Entre el 1 de marzo de 2002 y el 31 de abril de 2008, se trataron 342 pacientes con HSAa en nuestro hospital. De estos, 64 tenían una puntuación de 4 ó 5 de la escala de la WFNS (World Federation of Neurosurgical Societies). En el presente estudio prospectivo se analizan 11 pacientes en los que se realizó una CDP durante la misma cirugía de clipaje o/y evacuación del hematoma asociado.

Resultados. En tres pacientes la CDP se realizó después del tratamiento endovascular del aneurisma debido a la necesidad de evacuar el hematoma asociado. En los ocho pacientes restantes, la CDP se realizó durante el clipaje y la evacuación del hematoma asociado. La evaluación de los resultados de estos once pacientes se llevó a cabo al año de la cirugía mediante la escala de resultados de Glasgow. La CDP fue eficaz en el control de presión intracraneal en los pacientes que sobrevivieron. Seis pacientes sobrevivieron, cuatro de ellos con un buen resultado neurológico. Sin embargo, dos de estos seis pacientes tuvieron un resultado desfavorable.

De los cinco pacientes que no sobrevivieron, un paciente murió a causa de un hematoma subgalealepidural diferido como complicación de la CDP, y los restantes cuatro pacientes fallecieron a causa de una

Recibido: 7-04-10. Aceptado: 9-08-10 hipertensión intracraneal refractaria.

Conclusiones. La CDP puede ser una alternativa terapéutica en un grupo seleccionado de pacientes con HSAa con mal grado neurológico. Sin embargo, en la actualidad hay una falta de evidencia para apoyar unas recomendaciones claras para su uso.

PALABRAS CLAVE: Hemorragia subaracnoidea aneurismática. Craniectomía descompresiva. Craniectomía descompresiva profiláctica. Hematoma intracerebral.

Primary decompressive craniectomy in patients with aneurysmatic subarachnoid hemorrhage. Results of a pilot study in 11 cases

Summary

Introduction. Despite the scientific and technical advances of recent years, aneurysmal subarachnoid hemorrhage (aSAH) continues to present a high morbidity and mortality. This fact, together with the impressive results of the primary decompressive craniotomy (PDC) in the malignant infarction of the middle cerebral artery suggests a possible beneficial effect of decompressive technique in aSAH. We present our experience of a pilot study that PDC was used in patients with poorgrade aSAH with associated intracerebral hematoma.

Patients and methods. Between March $1^{\text {st }}, 2002$ and $31^{\text {st }}$ April, 2008, 342 patients with aneurysmatic subarachnoid hemorrhage (aSAH) were treated at our hospital. Of these, 64 had a poor neurological grade (scores of 4 or 5 of the World Federation of Neurosurgical Societies) at the time of admission. The present study exami-

Abreviaturas. aSAH: aneurysmal subarachnoid hemorrhage. CD: craniectomía descompresiva. CDP: craneotomía descompresiva primaria. CDS: craneotomía descompresiva secundaria (CDS) HSAa: hemorragia subaracnoidea aneurismática. IMACM: infarto maligno de la arteria cerebral media. PDC: primary decompressive craniotomy. PIC: presión intracranial. WFNS: World Federation of Neurosurgical Societies. 
nes 11 of those patients who underwent PDC, which is performed in the same clipping and / or evacuation of an associated hematoma.

Results. In three patients PDC was performed after endovascular aneurysm treatment because of the need to evacuate an associated hematoma. In the eight remaining patients, PDC was performed in the same clipping and evacuation of the associated hematoma. Outcome evaluation of these eleven patients was conducted 1 year after the operation assessed by the Glasgow Outcome Scale. Six patients survived, and four of them with good results. The PDC was effective in controlling intracranial pressure in all six surviving patients. However, two of these six patients had unfavorable outcomes.

Of the five who didn't survive, one patient died from a delayed epidural-subgaleal hematoma as a complication of the decompressive technique, and the other four patients died because of refractory intracranial hypertension.

Conclusions. Primary DC may be beneficial in selected subgroups of patients with poor-grade aSAH. However, there is a lack of definitive evidence to support a clear recommendation for its use.

KEY WORDS: Aneurysmatic subarachnoid hemorrhage. Decompressive craniectomy. Prophylactic decompressive craniectomy. Intracerebral hematoma.

\section{Introducción}

Tras la primera descripción por Kocher en $1901^{11}$, han existido periodos de escepticismo y otros de auténtico entusiasmo ante la craniectomía descompresiva (CD). El soporte más importante a esta técnica se ha dado tras la publicación de la eficacia incontrovertida de esta técnica en reducir la mortalidad en el infarto maligno de la arteria cerebral media (IMACM) 20,21 .

Actualmente, el traumatismo craneoencefálico, el IMACM y la hemorragia subaracnoidea son las principales áreas de estudio en la eficacia e indicaciones de la CD. En una revisión sistemática reciente se ha diferenciado entre dos tipos de CD: la primaria y la secundaria ${ }^{17}$. La craniectomía descompresiva primaria (CDP), también denominada profiláctica, se define como cualquier descompresión quirúrgica realizada con o sin extirpación de tejido cerebral en pacientes intervenidos principalmente para la evacuación de una lesión intracraneal de cualquier tipo. El objetivo de la CDP no es controlar una presión intracraneal (PIC) refractaria, sino la de evitar previsibles aumentos postquirúrgicos de la PIC, o bien para ayudar a su control en pacientes que ya están sometidos a monitorización de la PIC y que requieren cirugía intracraneal. En estos procedimientos, la decisión la toma el neurocirujano, independiente a la PIC, y normalmente se basa en datos de neuroimagen y/o en hallazgos intraoperatorios (tumefacción cerebral o incluso la dificultad para la reposición del colgajo óseo).

La craniectomía descompresiva secundaria (CDS) o terapéutica, es aquélla realizada para controlar la PIC elevada refractaria al tratamiento médico. Esta opción terapéutica se utiliza en algunos centros tras el fracaso de las medidas de primer y segundo nivel en el control de la PIC. El uso de la CDP en el tratamiento o prevención de la hipertensión intracraneal secundaria a la hemorragia subaracnoidea ha recibido menor atención que en el IMACM o en el traumatismo craneoencefálico. Esto queda reflejado claramente en la diferencia en el número de publicaciones existentes en las distintas áreas.

A pesar de los avances científicos y técnicos de los últimos años, la hemorragia subaracnoidea aneurismática (HSAa) sigue presentando una elevada morbilidad y mortalidad ${ }^{5,14,15}$. Este hecho, junto con los espectaculares resultados de la CD en el IMACM sugiere el potencial efecto beneficioso de la técnica descompresiva en este tipo de pacientes.

Presentamos nuestra experiencia con la CDP en un estudio piloto realizado en pacientes con HSAa con un mal grado neurológico y un hematoma asociado, en los que se realizó la CD profiláctica tras el clipaje y la evacuación del hematoma o bien después de la evacuación quirúrgica de un hematoma asociado después del tratamiento endovascular del aneurisma.

\section{Pacientes y métodos}

Entre el 1 de marzo 2002 y el 31 de abril de 2008, se trataron en nuestro hospital 342 pacientes con HSAa. De estos, 64 tenían un mal grado neurológico en el momento del ingreso (puntuaciones de 4 o 5 de la escala de la WFNS). Diecisiete de estos 64 pacientes presentaban además un hematoma intracerebral o silviano superior a $25 \mathrm{cc}$. El presente estudio analiza los resultados de 11 pacientes tratados con una CDP, realizada durante la misma cirugía de clipaje y/o evacuación del hematoma asociado, al considerar el cirujano que esta técnica podía ofrecer un potencial beneficio clínico para el control de la PIC postoperatoria.

\section{Datos clínicos}

Todos los datos clínicos, radiológicos y de resultados fueron registrados en una base de datos específica de la unidad de neurocirugía vascular creada en Microsoft Excel versión XP (Microsoft Corporation, Washington, Estados Unidos) específicamente para los pacientes con patología aneurismática cerebral.

\section{Medición del Hematoma}

Para la medición del componente hiperdenso de la le- 
sión intradural se siguió el método denominado $\mathrm{ABC} / 2$, fundamentado en el cálculo del volumen de un elipsoide ${ }^{1,12}$, de acuerdo con la siguiente ecuación: $\mathrm{V}=\left(4 \prod \mathrm{abc}\right) / 3$, donde $\mathrm{a}, \mathrm{b}$ y c corresponden a los radios del elipsoide. Para la simplificación del cálculo, se sustituyen los radios por sus respectivos diámetros ( $\mathrm{A}, \mathrm{B}$ y $\mathrm{C}$ ), la suma se divide por 2 y se aproxima el valor de $\Pi$ a 3 , lo que permite expresar la fórmula de la siguiente manera: Volumen $=4 / 3(3)(\mathrm{A} / 2 \mathrm{~B} / 2$ $\mathrm{C} / 2)=4(\mathrm{ABC} / 8)=\mathrm{ABC} / 2$.

Para la aplicación de este método se seleccionó el corte de la TC cerebral donde se observó el área hemorrágica de mayor tamaño, siendo A el diámetro máximo anteroposterior del área hiperdensa y $\mathrm{B}$ el diámetro transversal perpendicular a $\mathrm{A}$ en el mismo corte. El parámetro $\mathrm{C}$ o altura de la lesión se calculó mediante la substracción de los valores de la posición de la camilla del último y del primer corte en los cuales se visualizaba la lesión hiperdensa.

\section{Protocolo hospitalario}

Todos los pacientes fueron tratados siguiendo el protocolo institucional para la HSAa, consensuado por el equipo de patología cerebrovascular de nuestro hospital de acuerdo a las recomendaciones internacionales y a su adaptación a nuestro sistema de salud. Tras la admisión del paciente y su reanimación, y una vez demostrado por TC el diagnóstico de HSA, el estado neurológico siempre fue valorado por alguno de los neurocirujanos del servicio, considerando el volumen del hematoma superior a $25 \mathrm{cc}$ como criterio de cirugía urgente.

La técnica de la exclusión del aneurisma (quirúrgica o endovascular) fue elegida en función de la ubicación y de las características angio-arquitectónicas del aneurisma (tamaño y relación saco-cuello). En general, se excluyen quirúrgicamente los aneurismas de circulación anterior con una relación saco-cuello $\geq 2$, aneurismas muy pequeños $(\leq 2 \mathrm{~mm})$, grandes y gigantes de circulación anterior y aquellos aneurismas en que no ha sido factible el tratamiento endovascular. En aquellos casos en los que por la situación clínica del paciente no puede demorarse la cirugía evacuadora, y siempre que se visualice correctamente el aneurisma mediante un estudio angio-TC, la exclusión del aneurisma se realiza mediante clipaje quirúrgico omitiendo el estudio angiográfico.

\section{Procedimiento quirúrgico}

En los casos en los que se decidió la exclusión endovascular del aneurisma, la cirugía fue realizada inmediatamente después de la embolización del aneurisma, trasladando al paciente desde la sala de neurorradiología vascular al quirófano de neurocirugía. En estos casos, la cirugía se planificó para evacuar el hematoma y realizar una amplia hemicraniectomía descompresiva.

Cuando por las características del aneurisma se realizó un clipaje quirúrgico, el paciente fue trasladado inmediatamente al quirófano. Mediante una incisión cutánea tipo question mark se realizó una craneotomía pterional extendida ampliamente hacia las regiones frontal, temporal y parietal, con el fin de poder realizar en el mismo acto, el clipaje microquirúrgico del aneurisma, la evacuación del hematoma y la hemicraniectomía descompresiva. El colgajo óseo fue transferido a un banco de tejidos en donde se congeló a $-80^{\circ} \mathrm{C}$ para su conservación y posterior reposición.

La duramadre se abrió de forma semicircular con incisiones adicionales para permitir la máxima descompresión. Para conseguir la mayor relajación del cerebro durante el clipaje microquirúrgico y/o la evacuación del hematoma, se recurrió a la diuresis osmótica (manitol $1-2 \mathrm{mg} / \mathrm{kg}$ ) y al drenaje de líquido cefalorraquídeo. Al cierre, se realizó una plastia de duramadre utilizando en todos los casos un sustituto biológico de pericardio bovino (Tutopatch ${ }^{\circledR}$, Tutogen Medical, Neunkirchen, Alemania). Los márgenes de la duramadre fueron suspendidos a los bordes de la craneotomía para evitar la formación de colecciones o hematomas epidurales-subgaleales. La reconstrucción craneal se efectuó en el plazo de uno a dos meses.

Tras la cirugía, todos los pacientes fueron tratados en la unidad de cuidados intensivos para evitar o controlar la hipertensión intracraneal y el vasoespasmo, mediante la monitorización de la PIC y la evaluación diaria del Doppler transcraneal. La monitorización continua de la PIC se realizó mediante un sensor intraparenquimatoso (Integra Neurocare, Plainsboro, NJ, USA) colocado en el lado contralateral a la craniectomía. En presencia de vasoespasmo confirmado por angio-TC, arteriografía cerebral o doppler transcraneal (velocidades medias a nivel de la arteria cerebral media superiores a $120 \mathrm{~cm} / \mathrm{s}$ e índice Lindegaard superior a 3), y una vez excluido el aneurisma, el objetivo del tratamiento consistió en incrementar la presión arterial sistólica entre un $10-15 \%$ de la presión arterial basal del paciente (hipertensión moderada) mediante el uso de drogas vasopresoras (noradrenalina/fenilefrina) y evitando al mismo tiempo la sobrecarga de fluidos que pudiesen ocasionar una situación de hipervolemia. En caso de hipertensión intracraneal y en ausencia de nuevas lesiones hemáticas ocupantes de espacio, se inició siempre un tratamiento escalonado de la hipertensión intracraneal que incluía, como medidas terapéuticas de primer nivel, la administración de relajantes musculares (vecuronio), la evacuación de líquido cefalorraquídeo, y la administración de bolus intermitentes de soluciones hiperosmolares (manitol o suero salino hipertónico al 7,2\%) evitando siempre las situaciones de hipovolemia que pudiesen facilitar los déficits isquémicos.

\section{Evaluación de los resultados neurológicos}

La evaluación neurológica final se llevó a cabo un año 
Tabla 1

Caracteríscticas clínicas y radiológicas de los pacientes

\begin{tabular}{|c|c|c|c|c|c|c|c|c|c|}
\hline $\mathrm{N}^{o}$ & Edad /Sexo & WFNS & $\begin{array}{l}\text { Localiza- } \\
\text { ción del } \\
\text { aneurisma }\end{array}$ & $\begin{array}{c}\text { Volumen } \\
\text { del } \\
\text { hematoma } \\
(\mathrm{ml})\end{array}$ & $\begin{array}{l}\text { Desplaza- } \\
\text { miento de } \\
\text { la línea } \\
\text { media }(\mathrm{mm})\end{array}$ & $\begin{array}{l}\text { Tratamiento } \\
\quad \text { de } \\
\text { exclusión del } \\
\text { aneurisma }\end{array}$ & $\begin{array}{l}\text { Lateralidad } \\
\text { de la } \\
\text { craneotomía }\end{array}$ & $\begin{array}{l}\text { GOS } \\
\text { al alta }\end{array}$ & $\begin{array}{l}\text { GOS } \\
\text { al año }\end{array}$ \\
\hline 1 & $\pi / 46$ & 5 & $\mathrm{ACM}$ & 67 & 3 & Clip & Derecha & 1 & 1 \\
\hline 2 & $q / 38$ & 5 & $\mathrm{ACM}$ & 54 & 5 & Clip & Derecha & 1 & 1 \\
\hline 3 & q $/ 36$ & 4 & $\mathrm{ACM}$ & 60 & 13 & Clip & Izquierda & 4 & 4 \\
\hline 4 & $q / 40$ & 5 & ACOP & 27 & 1 & Coil & Izquierda & 4 & 5 \\
\hline 5 & $+/ 36$ & 4 & $\mathrm{ACM}$ & 40 & 4 & Coil & Derecha & 5 & 5 \\
\hline 6 & $\delta / 20$ & 5 & $\mathrm{ACM}$ & 70 & 9 & Coil & Derecha & 1 & 1 \\
\hline 7 & $ふ / 60$ & 4 & $\mathrm{ACM}$ & 60 & 6 & Clip & Derecha & 3 & 4 \\
\hline 8 & $9 / 43$ & 5 & $\mathrm{ACM}$ & 71 & 10 & Clip & Izquierda & 1 & 1 \\
\hline 9 & $q / 54$ & 4 & $\mathrm{ACM}$ & 76 & 7 & Clip & Izquierda & 2 & 2 \\
\hline 10 & $9 / 69$ & 5 & $\mathrm{ACM}$ & 67 & 7 & Clip & Derecha & 1 & 1 \\
\hline 11 & P/50 & 5 & $\mathrm{ACM}$ & 41 & 2 & Clip & Izquierda & 3 & 3 \\
\hline
\end{tabular}

ACOP indica aneurisma de la arteria comunicante posterior y ACM aneurisma de la arteria cerebral media

después de la hemorragia, mediante la Escala de Resultados de Glasgow (GOS), que consta de 5 niveles: buena recuperación, discapacidad moderada, grave discapacidad, estado vegetativo y la muerte. El GOS se dicotomizó en resultados favorables (buena recuperación y discapacidad moderada) y desfavorables ${ }^{7}$ (discapacidad grave, estado vegetativo y muerte).

\section{Análisis estadístico}

Las variables recogidas en este estudio se introdujeron en una base de datos específica para su análisis final mediante el programa estadístico SPSS v.16.0 (SPSS Inc., Chicago, IL, EEUU) Se realizó un análisis descriptivo de las variables cuantitativas expresándose los resultados mediante la media \pm desviación típica. Para comparar los resultados neurológicos entre los grupos de pacientes se aplicó la prueba exacta de Fisher. El nivel de significación estadística se estableció para una $\mathrm{P} \leq 0,05$.

\section{Resultados}

Ocho pacientes fueron mujeres y tres hombres. La edad media de la serie fue de $45 \pm 13$ años (rango, 20-69). Según su estado neurológico, cuatro pacientes fueron clasificados al ingreso en nuestro centro con un grado IV de la WFNS y siete con un grado V. Las características clínicas y radiológicas de los pacientes se resumen en la Tabla 1.

La medición del volumen del hematoma en la TC de ingreso mostró un volumen medio de 57,5 $\pm 15,5 \mathrm{ml}$ y un desplazamiento de la línea media de $6,1 \pm 3 \mathrm{~mm}$. A excepción de un paciente con un aneurisma situado en la arteria comunicante posterior, el resto de los pacientes presentaban un aneurisma localizado en la arteria cerebral media.

En tres pacientes, la CDP se llevó a cabo después del tratamiento endovascular del aneurisma, debido a la necesidad de evacuación del hematoma asociado. En los ocho pacientes restantes, la CDP se realizó en el mismo acto de clipaje y evacuación del hematoma asociado. Antes 


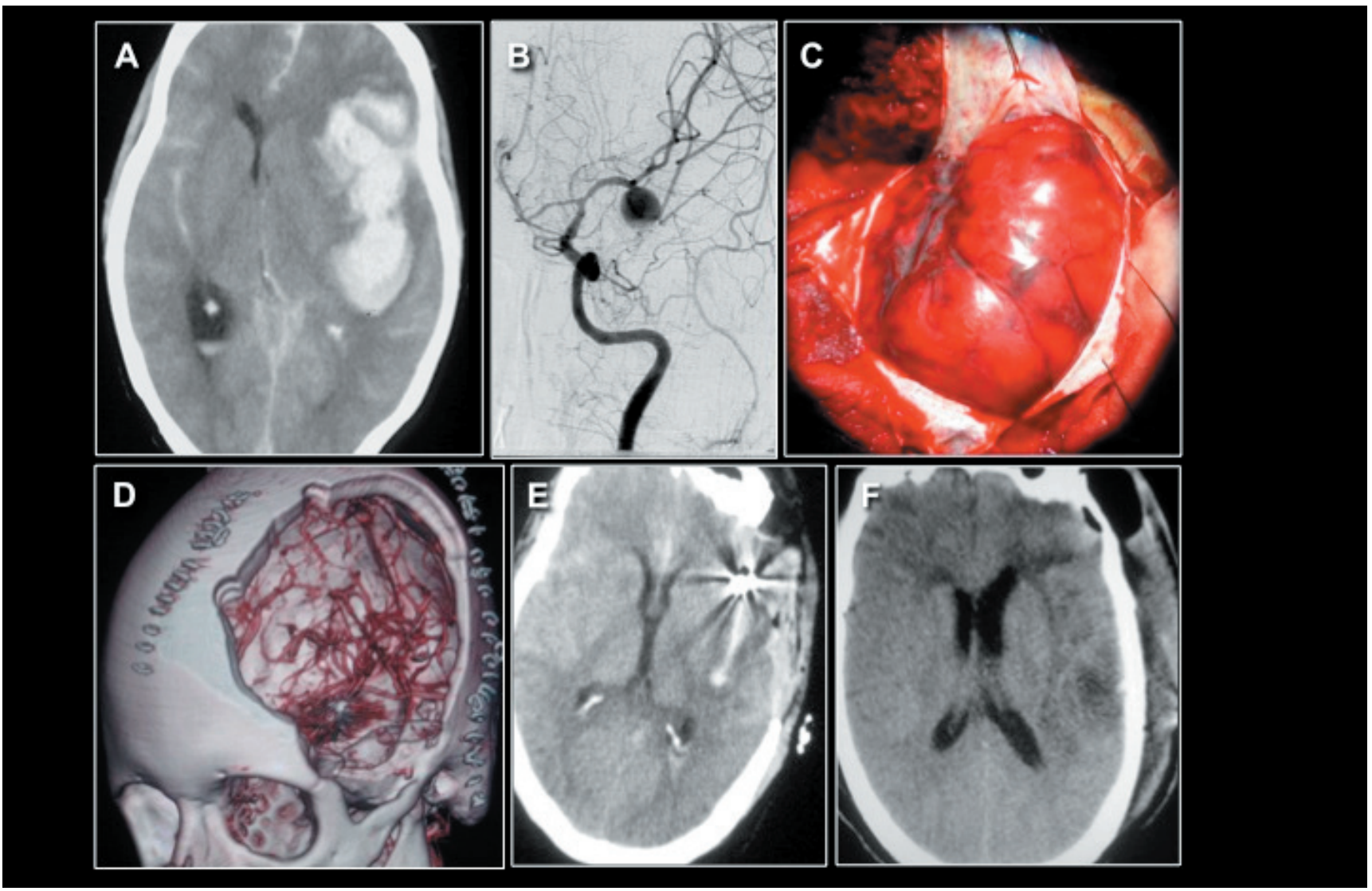

Figura 1. Ejemplo del paciente número 3. Mujer de 36 años de edad, encontrada inconsciente en su domicilio. La exploración neurológica realizada por el Servicio de Urgencias Medicas destaca una puntuación de 8 en la Escala de Coma de Glasgow y una hemiparesia derecha. La TC craneal realizada de urgencias revela la presencia de una hemorragia subaracnoidea asociada con un gran hematoma cuantificado en 60 cc (Imagen A). La angiografía diagnosticó un aneurisma sacular situado en la arteria cerebral media izquierda (Imagen B). El paciente fue operado de urgencias; dados los hallazgos clínicos y radiológicos se realizó una craneotomía pterional extendida ampliamente hacia las regiones frontal, temporal y parietal, con el fin de poder realizar en el mismo acto el clipaje microquirúrgico del aneurisma, la evacuación del hematoma y la hemicraniectomía descompresiva (Imagen C). La imagen D muestra una reconstrucción tomográfica de la craniectomía descompresiva realizada. La imagen E muestra la TC craneal postoperatoria y la imagen F la TC craneal realizada tras la reconstrucción craneal. El paciente presentó tanto en el momento del alta como en el control anual una moderada discapacidad.

de la cirugía, cinco de estos ocho pacientes fueron evaluados únicamente mediante angiografía por tomografía computarizada (sin angiografía por sustracción digital) debido a la urgencia y la correcta visualización del aneurisma responsable de la hemorragia.

En seis pacientes se realizó una hemicraniectomía derecha y en los cinco restantes izquierda.

La CDP fue eficaz en el control de la PIC en los seis pacientes supervivientes. La evaluación final de los resultados de estos once pacientes se llevó a cabo al año de la HSAa. Seis pacientes sobrevivieron, cuatro de ellos con buenos resultados (dos obtuvieron una buena recuperación y dos una discapacidad moderada). Dos de los seis pacientes tuvieron un resultado desfavorable, uno con una discapacidad grave y otro que permanecía en un estado vegetativo en el control al año.

En cuatro de los cinco pacientes que murieron la CDP no consiguió controlar la PIC, siendo la hipertensión intracraneal la causa de la muerte. El quinto paciente falleció de un hematoma epidural-subgaleal diferido como una complicación de la CDP. Clínicamente la paciente se encontraba consciente y parcialmente desorientada, presentando una leve hemiparesia izquierda. No hubo otras complicaciones de la CD en los otros diez pacientes. En función del grado neurológico según la escala de la WFNS, tres de los cuatro pacientes con un grado IV alcanzaron un buen resultado, mientras que sólo uno de los siete en grado V logró un resultado favorable. Sin embargo, pro- 


\section{Tabla 2}

Estado neurológico del paciente al ingreso según la escala de la Federación Mundial de Neurocirujanos (WFNS) y resultado al año según la escala de resultados de Glasgow (GOS)

\begin{tabular}{ccccc}
\hline & Grado IV & Grado V & Total \\
\hline $\mathbf{N}^{\mathbf{0}}$ pacientes & & 4 & 7 & 11 \\
GOS & $\mathbf{R F}(\mathbf{B R}, \mathbf{M D})$ & 3 & 1 & 4 \\
& MR (SD, EV. M) & 1 & 6 & 7
\end{tabular}

$\mathrm{RF}=$ Resultado favorable; $\mathrm{BR}=$ Buena Recuperación; $\mathrm{MD}=$ Moderada discapacidad; $\mathrm{MR}=\mathrm{Mal}$ Resultado; $\mathrm{SD}=\mathrm{Severa}$ Discapacidad; $\mathrm{EV}=$ Estado Vegetativo and $\mathrm{M}=$ Muerte

Test exacto de Fisher's $\mathrm{p}=0,088$

bablemente debido al escaso número de pacientes en este estudio piloto, el estado neurológico no alcanzó una significación estadística en el pronóstico final de estos pacientes (Tabla 2). Tampoco el hemisferio afectado fue un factor predictivo, debido de nuevo probablemente al reducido tamaño muestral.

\section{Discusión}

La HSAa es una entidad que representa alrededor del $5 \%$ de los accidentes vasculares cerebrales. Es bien conocido que uno de los factores pronósticos más decisivos en la hemorragia subaracnoidea es el estado neurológico inicial del paciente, de modo que los pacientes con hemorragias subaracnoideas que debutan con un mal grado neurológico presentan peores resultados clínicos que los que presentan un buen estado neurológico ${ }^{14}$. En el primer grupo la mortalidad puede llegar a ser del $98 \%{ }^{22}$.

Los principales problemas que ensombrecen el pronóstico de la HSAa, son la hipoxia tisular isquémica secundaria a vasoespasmo ${ }^{9}$ y la hipertensión intracraneal ${ }^{4,10,13,14}$. Estas dos complicaciones siguen siendo en la actualidad las principales causas de morbilidad y mortalidad, puesto que el tratamiento precoz de la exclusión del aneurisma ha reducido de forma muy significativa el riesgo de resangrado.

Sin embargo, la hipertensión intracraneal secundaria supone un problema clínico de difícil manejo, puesto que la deshidratación que conlleva el tratamiento osmótico o la vasoconstricción de la hiperventilación, se contraponen con el tratamiento del vasoespasmo. Así pues, la necesidad de avances en el tratamiento de estos pacientes, junto con los espectaculares resultados obtenidos por la $\mathrm{CD}$ en el IMACM $^{20,21}$ está despertando de forma progresiva el interés de esta técnica en los pacientes con una HSAa.

$\mathrm{Al}$ igual que en el traumatismo craneoencefálico o en el IMACM, el fundamento de la craniectomía descompresiva en la HSAa es la de prevenir o controlar el aumento de la PIC, mejorar la presión de perfusión cerebral, prevenir las lesiones isquémicas, mejorar la circulación colateral leptomeníngea y evitar las compresiones mecánicas sobre estructuras cerebrales producidas por herniaciones cerebra$\operatorname{les}^{6,8,19}$.

Tal como ha demostrado Bailes ${ }^{2}$, en los pacientes con hemorragia subaracnoidea en mal grado neurológico y sin evidencia de lesiones cerebrales irreversibles, el manejo clínico rápido y efectivo de la presión intracraneal se asocia a mejores resultados.

La craniectomía descompresiva puede ser realizada en dos situaciones muy diferentes. La primera es la denominada craniectomía profiláctica o descompresiva primaria, que en el caso de la patología aneurismática sería aquélla descompresiva realizada en el mismo acto quirúrgico de clipaje o/y evacuación de un hematoma asociado, al considerar el cirujano esta técnica beneficiosa para el control postoperatorio de la presión intracraneal. Así pues, el objetivo de la CDP no es el tratamiento de una hipertensión intracraneal refractaria, sino que la decisión realizada por el cirujano suele basarse en los datos clínicos, radiológicos $\mathrm{o} / \mathrm{y}$ intraoperatorios que hacen prever una hipertensión intracraneal de difícil control postoperatorio o incluso motivada por la imposibilidad de reponer el colgajo óseo debido a la presencia de un cerebro tumefacto que se hernia a través de los márgenes de la craneotomía ${ }^{17}$.

En este sentido, Ogilvy et al. publicaron en 2002 los resultados en el uso de la CDP en pacientes en mal grado neurológico con HSAa asociadas a hematomas por la rotura de aneurisma localizados a nivel de la bifurcación de la arteria cerebral media ${ }^{18}$. Este trabajo, publicado tan solo hace seis años, constituye el primer estudio dirigido a demostrar la eficacia de la CD profiláctica en el contexto de la hemorragia subaracnoidea. En este estudio prospectivo se incluyeron 8 pacientes con aneurismas de la arteria 
cerebral media asociados a hematomas de volumen superior a $25 \mathrm{ml}$ (media $121 \mathrm{ml}$ ) en los que se realizó una CDP durante la cirugía de clipaje y evacuación del hematoma. El buen control de la PIC, así como los excelentes resultados clínicos obtenidos a los 12 meses, permitieron concluir a sus autores que la craniectomía descompresiva constituía una técnica que podía beneficiar a un subgrupo bien seleccionado de pacientes con hemorragias subaracnoideas.

Sin embargo, como ha ocurrido en otras áreas de estudio, tras el optimismo inicial, apareció pocos años después un estudio realizado por Ambrosio et al. que mostraban que si bien la CD reducía de forma significativa la mortalidad, los supervivientes mostraban unos malos resultados en las escalas de calidad de vida ${ }^{3}$.

Otani et al. han publicado recientemente una serie de 57 pacientes con HSAa asociada a hematoma en mal grado neurológico estudiados de forma retrospectiva ${ }^{16}$. Estos autores observaron un buen resultado neurológico en el $37 \%$ de los casos, sugiriendo que la CD podría asociarse a una tasa aceptablemente baja de malos resultados en este subgrupo de pacientes.

Nuestra experiencia es similar a este último trabajo. Nuestros resultados sugieren que las CDP podría ser beneficiosa en el subgrupo de pacientes con una HSAa asociada a hematoma intracerebral, en mal grado neurológico y sin evidencia de lesión neurológica irreversible.

Sin embargo, nuestro estudio tiene limitaciones importantes. En primer lugar, es un análisis retrospectivo y como tal, nuestras observaciones deben ser validadas de forma prospectiva. En segundo lugar, el tamaño muestral de nuestro estudio es pequeño, lo que limita el análisis estadístico. Debemos considerar que el Hospital Universitario Vall d'Hebron es uno de los hospitales terciarios con un mayor volumen de patología vascular en Cataluña, y a pesar de ello, obtener esta muestra ha requerido un largo período de tiempo. Por ello, la confirmación de nuestros resultados debería verificarse en un estudio multicéntrico controlado con asignación aleatoria del tratamiento.

\section{Conclusiones}

La CD primaria puede ser beneficiosa en grupos seleccionados de pacientes con HSAa en mal estado neurológico. Sin embargo, hay una falta de estudios concluyentes para apoyar una recomendación clara para su uso. La confirmación de nuestros resultados debería verificarse en un estudio multicéntrico controlado con asignación aleatoria del tratamiento.

\section{Bibliografía}

1. Arikan, F., Sahuquillo, J., Ibañez, J., et al: [Variability in the surgical indications for posttraumatic intradural lesions.].
Neurocirugia. 2005; 16: 108-116.

2. Bailes, J.E., Spetzler, R.F., Hadley, M.N., Baldwin, H.Z.: Management morbidity and mortality of poor-grade aneurysm patients. J Neurosurg 1990; 72: 559-566.

3. D'Ambrosio, A.L., Sughrue, M.E., Yorgason, J.G., et al: Decompressive hemicraniectomy for poor-grade aneurysmal subarachnoid hemorrhage patients with associated intracerebral hemorrhage: clinical outcome and quality of life assessment. Neurosurgery 2005; 56: 12-19; dicussion 19-20.

4. Hayashi, M., Kobayashi, H., Kawano, H., Yamamoto, S., Maeda, T.: Cerebral blood flow and ICP patterns in patients with communicating hydrocephalus after aneurysm rupture. J Neurosurg 1984; 61: 30-36.

5. Hop, J.W., Rinkel, G.J., Algra, A., van Gijn, J.: Casefatality rates and functional outcome after subarachnoid hemorrhage: a systematic review. Stroke 1997; 28: 660-664.

6. Jaeger, M., Soehle, M., Meixensberger, J.: Effects of decompressive craniectomy on brain tissue oxygen in patients with intracranial hypertension. J Neurol Neurosurg Psychiatry 2003; 74: 513-515.

7. Jennett, B., Bond, M.: Assessment of outcome after severe brain damage. Lancet 1975; 1: 480-484.

8. Jourdan, C., Convert, J., Mottolese, C., Bachour, E., Gharbi, S., Artru, F.: [Evaluation of the clinical benefit of decompression hemicraniectomy in intracranial hypertension not controlled by medical treatment]. Neurochirurgie 1993; 39: 304-310.

9. Kassell, N.F., Sasaki, T., Colohan, A.R., Nazar, G.: Cerebral vasospasm following aneurysmal subarachnoid hemorrhage. Stroke 1985; 16: 562-572.

10. Kaye, A.H., Brownbill, D.: Postoperative intracranial pressure in patients operated on for cerebral aneurysms following subarachnoid hemorrhage. J Neurosurg 1981; 54: 726-732.

11. Kocher, T., Schlesinger, E.B.: Hirnerschütterung, Hirndruck und chirurgische Eingriffe bei Hirnkrankheiten. Wien 1991: Alfred Hölder.

12. Kothari, R.U., Brott, T., Broderick, J.P., et al: The $\mathrm{ABCs}$ of measuring intracerebral hemorrhage volumes. Stroke 1996; 27: 1304-1305.

13. Le Roux, P.D., Elliott, J.P., Downey, L., et al: Improved outcome after rupture of anterior circulation aneurysms: a retrospective 10-year review of 224 good-grade patients. J Neurosurg 1995; 83: 394-402.

14. Le Roux, P.D., Elliott, J.P., Newell, D.W., Grady, M.S., Winn, H.R.: Predicting outcome in poor-grade patients with subarachnoid hemorrhage: a retrospective review of 159 aggressively managed cases. J Neurosurg 1996; 85: 39-49.

15. Molyneux, A.J., Kerr, R.S., Yu, L.M., et al: International subarachnoid aneurysm trial (ISAT) of neurosurgical clipping versus endovascular coiling in 2143 patients with ruptured intracranial aneurysms: a randomised comparison of effects on survival, dependency, seizures, rebleeding, sub- 
groups, and aneurysm occlusion. Lancet 2005; 366: 809-817.

16. Otani, N., Takasato, Y., Masaoka, H., et al: Surgical Outcome following Decompressive Craniectomy for PoorGrade Aneurysmal Subarachnoid Hemorrhage in Patients with Associated Massive Intracerebral or Sylvian Hematomas. Cerebrovasc Dis 2008; 26: 612-617.

17. Sahuquillo, J., Arikan, F.: Decompressive craniectomy for the treatment of refractory high intracranial pressure in traumatic brain injury. Cochrane.Database.Syst.Rev. 2006; CD003983

18. Smith, E.R., Carter, B.S., Ogilvy, C.S.: Proposed use of prophylactic decompressive craniectomy in poor-grade aneurysmal subarachnoid hemorrhage patients presenting with associated large sylvian hematomas. Neurosurgery 2002; 51: 117-124.

19. Stiefel, M.F., Heuer, G.G., Smith, M.J., et al: Cerebral oxygenation following decompressive hemicraniectomy for the treatment of refractory intracranial hypertension. J.Neurosurg 2004; 101: 241-247.

20. Vahedi, K.: Decompressive hemicraniectomy for

Comentarios al trabajo Craniectomía descompresiva primaria en la hemorragia subaracnoidea aneurismática. Resultados de un estudio piloto en 11 casos de F Arikan y cols

Los autores presentan su experiencia con la craniectomía descompresiva primaria en el manejo de los pacientes con HSA en mal grado neurológico y con un hematoma intracerebral o silviano mayor de $25 \mathrm{cc}$ que debe de ser evacuado, aprovechando la cirugía urgente para vaciar el hematoma, clipar el aneurisma (salvo en los casos previamente embolizados) y dejar la craniectomía hecha de forma profiláctica para prevenir una posible hipertensión intracraneal.

Los buenos resultados de la craniectomía descompresiva primaria o profiláctica en casos seleccionados de traumatismo y de infarto maligno de la arteria cerebral media, han hecho plantearse a los autores su extensión a este subgrupo de pacientes con HSA, apoyados por los aceptables resultados de los artículos publicados por el grupo de Massachusets de Ogilvy et al y el grupo japonés de Otani et al.

Teóricamente esta modalidad de tratamiento intentaría evitar la morbimortalidad añadida de una hipertensión intracraneal (HTIC) sobre los daños cerebrales ya producidos por la propia HSA y por el hematoma asociado. La malignant hemispheric infarction. Curr Treat Options Neurol 2009; 11: 113-119.

21. Vahedi, K., Hofmeijer, J., Juettler, E., et al: Early decompressive surgery in malignant infarction of the middle cerebral artery: a pooled analysis of three randomised controlled trials. Lancet Neurol 2007; 6: 215-222.

22. Zubkov, Y.N.: Treatment of patients with intracranial arterial aneurysms in the haemorrhagic period. Neurol Res 1994; 16: 6-8.

Arikan, F.; Vilalta, J.; Romero, F.J.; Porta, I.; MartínezRicarte, F.R.; Sahuquillo, J.: Craniectomía descompresiva primaria en la hemorragia subaracnoidea aneurismática. Resultados de un estudio piloto en 11 casos. Neurocirugía 2010; 21: 452-460.

Correspondencia: Fuat Arikan. Servicio de Neurocirugía. Hospital Universitario Vall d'Hebron. Paseo Vall d'Hebron 119-129. 08035 Barcelona

E-mail: arikan@neurotrauma.net duda que nos surge es si es realmente necesario realizar una craniectomía descompresiva profiláctica en este subgrupo de pacientes o si sería suficiente con evacuar el hematoma para intentar evitar un posible aumento de la PIC, sobre todo en los casos en que el cerebro no presenta datos de HTIC tras la cirugía. Hay que tener en cuenta que, en el subgrupo estudiado, el mal grado clínico se debe no sólo a la propia HSA sino también al deterioro que causa el hematoma, por lo que en este grupo de pacientes la "malignidad" de la HSA puede estar sobredimensionada por la presencia del hematoma. Al evacuarse éste, sin necesidad de hacer descompresiva, puede mejorar la situación clínica del paciente. Por tanto, los buenos resultados podrían deberse en gran parte a la evacuación del hematoma y a lo mejor no tanto a la craniectomía descompresiva. Para valorar el peso de cada una de estas actuaciones sería interesante comparar la evolución final del grupo analizado frente a los pacientes con HSA en mal grado con hematomas $>25 \mathrm{cc}$ en los que sólo se evacuó el hematoma pero se repuso el hueso, y en cuántos de éstos se necesitó una descompresiva secundaria 
por HTIC.

En general, se trata de un artículo metodológicamente correcto y claro en su redacción, que aborda por primera vez en nuestro país el uso de la craniectomía descompresiva primaria en este subgrupo de pacientes con HSA con unos
$2010 ; 21: 452-460$

resultados aparentemente prometedores, aunque, al igual que en los otros artículos mencionados por los autores, se trata de un grupo pequeño para extraer conclusiones significativas.

\author{
A. de la Lama Zaragoza
}

$* * * * * * * * * * * * * * * * * * * * * * * * * * * * * * * * * * *$

Los autores hacen un estudio sobre la realización de una craniectomía descompresiva en pacientes con hemorragia subaracnoidea aneurismática con peor puntuación en la escala de la WFNS (grados 4 y 5). Hasta el momento, todos los intentos por obtener un pronóstico más favorable en este grupo de enfermos ha sido infructuoso, por lo cual todo intento de hacer algo en este sentido tiene que ser bienvenido.

A pesar de ser un estudio retrospectivo, los resultados apuntan a que una actuación rápida con craniectomía descompresiva para controlar la presión intracraneal puede ser un factor determinante en el pronóstico de algu- nos de estos pacientes con mala situación neurológica a su entrada en el hospital, sobre todo aquéllos que se encuentran en un grado 4 de la WFNS. Este último grupo parece ser el más idóneo y beneficiado en recibir este tipo de tratamiento.

Como indican los propios autores, lo ideal sería realizar un estudio prospectivo multicéntrico para un análisis adecuado del tema. A esta idea me adhiero y, además, animo encarecidamente a los investigadores para que esto sea llevada a cabo en un futuro próximo. 